\title{
Seismic vulnerability assessment on a territorial scale based on a Bayesian approach
}

\author{
S. Taffarel, G.P. Campostrini, L. Rosato, F. da Porto \& C. Modena \\ University of Padova, Department of Civil, Environmental and Architectural Engineering, 35131 Padova, \\ Italy
}

\begin{abstract}
Italian historical centres are mostly characterized by aggregate buildings. As defined by the Italian codes (Norme Tecniche per le Costruzioni 2008 and Circolare n. 617), the analysis of the most representative local mechanisms of collapse must be performed in order to assess their vulnerability. In this article, the out-of-plane local mechanisms of collapse analysis is implemented by applying a new method of analysis based on a probabilistic approach. Usually information which are necessary for the implementation of the local mechanisms analyses are affected by uncertainty or are missing, therefore in lots of cases it is only possible to hypothesize them on the basis of the other buildings information collected during the on-site survey. In this context, the implementation of a Bayesian approach allows to deduce buildings lacking information (i.e. wall thickness and interstorey height) starting from certain collected data (i.e. facades height). The historical centre of Timisoara (Romania) is selected as the case study for the implementation of this new method of analysis, given the extension of the on-site survey already carried out in the area (information about more than 200 structural units have been collected) and the seismic vulnerability assessment on an urban scale already performed by applying a traditional method. Results obtained by adopting the two approaches are then compared and a validation and a calibration of the new one is carried out.
\end{abstract}

\section{INTRODUCTION}

During the last decades, reduction and mitigation of historical centres seismic risk became a urgent topic. Italian bodies and authorities at various levels are currently working to define procedures facing this issue (D.M. 14/01/2008, Circolare n. 617/2009, D.P.C.M 09/02/2011).

A first clear step that demonstrates this acquired increased awareness is given by the implementation of procedures for the collection of buildings information by adopting automatic procedures. The need to create a database shared throughout the whole Italian territory (and institutions) becomes more and more urgent. Ministerial bodies and research centers can retrieve buildings information to perform predictive assessments having the aim of reducing seismic vulnerability and, consequently, seismic risk. In this regard, several initiatives have been undertaken having the aim of increasing this process. As an example, the Italian Department of Civil Protection (DPC), in collaboration with Laboratories University Network of seismic engineering (ReLUIS), is developing a survey form for the characterization of urban areas (CARTIS) to be implemented at the Italian territory scale. The Ministry of Cultural Heritage and Tourism (MIBACT) edited a circular, the so-called
Circolare n.15 ("Provisions on the protection of architectural heritage and seismic risk mitigation"), which defines an incremental approach for the improvement of the reliability of seismic risk maps and for the documentation of local interventions implemented in listed buildings (Circolare n. 15/2015). Designers involved in seismic improvement or extraordinary maintenance intervention are encouraged to provide "essential" construction information about analyzed buildings. Usually implemented intervention do not involve buildings in their entirety but a limited part of them, so data inserted in the form can be partial. In the first phase of the procedure, the low level of information about structures does not represent a problem: data can be inserted in a database, which can be gradually updated each time new information become available.

\section{DATA MANAGEMENT: THE ISSUE OF INFORMATION UNCERTAINTY}

The process of data collection is proving essential in order to acquire information about structural features and vulnerability elements of the Italian built heritage. These data can be used by different institutions 
for the evaluation of seismic vulnerability on a territorial scale.

Considering the purpose of a seismic vulnerability assessment on a territorial scale, implemented by adopting simplified procedures based on a limited number of geometrical and mechanical parameters (MIBAC 2011), even if data collection is carried out by adopting traditional methods (expeditious on-site surveys and analysis of buildings mainly from the outside), collected information may be uncertain or even incomplete. Assessments involving the study of a huge number of buildings require that surveys are carried out by adopting expeditious procedures limiting time and cost consuming.

In light of these considerations, it is therefore essential to define methodologies for the seismic vulnerability assessment on an urban scale taking into account the uncertainty or the lack of information. As an example, during on-site surveys the collection of data relating to walls thickness is not always possible, because its measurement requires a complete inspection of analyzed buildings. Similarly, buildings total height is easily measurable from the outside using economic and readily available instrumentation (eg. Laser distance meters) but interstorey heights can only be estimated.

It is therefore essential to adopt procedures allowing the assessment of buildings vulnerability by adopting probabilistic approaches rather than a deterministic ones (Modena 2014).

University of Padova (Department of Civil, Architectural and Environmental engineering) implemented a new procedure deriving unknown quantities starting from known data / parameters through a Bayesian approach. Obtained data can then be used to perform local mechanisms of collapse analyses on existing buildings (D.M. 14/01/2008, Circolare n.617/2009). The analysis of local mechanisms of collapse is a fundamental step for the study of existing buildings seismic behavior. Historical constructions are usually aggregate buildings, so a global behavior is not easily identifiable. On the contrary, they can be easily subdivided into macroelements, which react individually to seismic events, as a set of subsystems (D.M. 14/01/2008, § C 8.7.1.1 Circolare n.617/2009).

\section{DESCRIPTION OF THE PROBABILISTIC APPROACH}

In some contexts and for some specific studies, starting available data are limited but, at the same time, experience of researchers can make up for this lack of information. In this context, Bayes theorem finds its natural application, providing the updating of initial hypotheses related to experience by using data which will be available a posteriori. The procedure of formalization of already available knowledge is called elicitation. The Bayes theorem allows the updating of initial assumptions, represented by adopted probability density functions, by using information acquired in a second stage. The theorem enables the updating of initial parameters, represented by $\boldsymbol{\theta}$ vector $\left(\boldsymbol{\theta}=\left[\theta_{1}, \theta_{2} \ldots \theta_{\mathrm{n}}\right]\right)$ and affected by uncertainty, introducing new available data $\left(\mathbf{y}=\left[\mathrm{y}_{1}, \mathrm{y}_{2} \ldots \mathrm{y}_{\mathrm{m}}\right]\right)$. The procedure can be described by the following scheme:

$p(\theta) \rightarrow y \rightarrow p(\theta \mid y)$

If the process is conducted by adopting continuous random variables, Bayes theorem can be reported as follow:

$p(\theta \mid y)=\frac{p(\theta) p(y \mid \theta)}{\int p(\theta) p(y \mid \theta) d \theta}$

where $\mathrm{p}(\boldsymbol{\theta})=$ prior distribution; $\mathrm{p}(\mathbf{y} \mid \boldsymbol{\theta})=$ likelihood; $\mathrm{p}(\boldsymbol{\theta} \mid \mathbf{y})=$ posterior distribution.

Quantity $\int p(\theta) p(y \mid \theta) d \theta$ is a constant.

Acquiring new information the posterior probability density function (pdf) can be used as prior pdf in a new application of (1) obtaining a new posterior pdf; this process can be adopted iteratively each time new data become available. In order to solve (2), conjugate distributions are adopted. A prior distribution can be defined as a distribution conjugated to the adopted sample model (or equivalently to likelihood) if prior and posterior distributions are characterized by the same functional form (Liseo 2008). One of the most important statistical models is the normal one, in which data are hypothesized having a normal distribution. The adoption of a normal distribution as an approximation of the real one is often justified if the sample size is composed by a sufficiently large amount of data (Liseo 2008). The calculation of posterior distribution can be implemented by considering different initial conditions:

-unknown mean $(\mu)$ and known variance $\left(\sigma^{2}\right)$;

-unknown $\mu$ and $\sigma^{2}$.

Initially, $\sigma^{2}$ is considered as a known parameter and inference exclusively refers to $\mu$. This assumption is not restrictive, because it can be applied to the cases in which both $\sigma^{2}$ is known and $\mu$ and $\sigma^{2}$ are unknown. Likelihood can be represented by the formula:

$$
L(\mu)=\prod_{i=1}^{n}\left(\frac{1}{\sigma \sqrt{2 \pi}} \exp \left\{-\frac{\left(y_{i}-\mu\right)^{2}}{2 \sigma^{2}}\right\} \propto \exp \left\{-\frac{n(\bar{y}-\mu)^{2}}{2 \sigma^{2}}\right\}\right.
$$

where: $\mathrm{n}=$ samples number; $\bar{y}=\frac{1}{n} \sum_{i=1}^{n} y_{i}$. 
The last part of the expression consider only factors depending on $\mu$.

If both $\mu$ and $\sigma^{2}$ are unknown, likelihood can be described as:

$L\left(\mu, \sigma^{2}\right)=\prod_{i=1}^{n}\left(\frac{1}{\sigma \sqrt{2 \pi}} \exp \left\{-\frac{\left(y_{i}-\mu\right)^{2}}{2 \sigma^{2}}\right\}\right) \propto \frac{1}{\sigma^{n}} \exp \left\{-\frac{n(\bar{y}-\mu)^{2}}{2 \sigma^{2}}\right\}$

$\propto \frac{1}{\sigma^{n}} \exp \left\{-\frac{n}{2 \sigma^{2}}\left[s^{2}+(\bar{y}-\mu)^{2}\right]\right\}$

where: $s^{2}=\frac{1}{n-1} \sum_{i=1}^{n}\left(y_{i}-\bar{y}\right)^{2}$ is the sample variance.

Prior pdf $p(\theta)=p\left(\mu, \sigma^{2}\right)$ can be factorised as:

$$
p\left(\mu, \sigma^{2}\right)=p\left(\mu \mid \sigma^{2}\right) p\left(\sigma^{2}\right)
$$

If as prior distribution a normal-inverse-gamma function defined by 4 hyperparameters $\left(\mu_{0}, \mathrm{n}_{0}, v_{0}, \sigma_{0}{ }^{2}\right)$ is chosen, by adopting Bayes theorem the posterior distribution is a normal-inverse-gamma too, which is defined by $\left(\mu_{1}, \mathrm{n}_{1}, v_{1}, \sigma_{1}{ }^{2}\right)$ hyperparameters (Hoff 2009).

Hyperparameters can be described by the following formulas (Jackman 2009):

$\mu_{1}=\frac{n_{0} \mu_{0}+n \bar{y}}{n_{0}+n}$

$n_{1}=n_{0}+n$

$v_{1}=v_{0}+v$

$v_{1} \sigma_{1}^{2}=v_{0} \sigma_{0}^{2}+s^{2}(n-1)+\frac{n_{0} n}{n_{0}+n}\left(\mu_{0}-\bar{y}\right)^{2}$

Starting from the posterior normal-inverse-gamma function, a certain number of samples characterized by couples of values, which represents respectively $\mu$ and $\sigma^{2}$, can be extracted. Standard deviation values (obtained by the variance) and mean are then used in order to generate normal distributions; for each of them, the code extracts a value that can be used for analyses implementation.

This procedure can be adopted to derive unknown information, having surveyed data related to the analyzed case study as input parameters. At the same time, for each unknown parameter, a series of data must be available in order to set a prior pdf. These data can be referred to other buildings having features that are similar to analysed ones (as an example, they belong to the same predefined typology). If these data are not available or are very poor, a prior distribution based on experience gained by the researcher carrying out the analysis can be assumed.
Input parameters adopted for the creation of prior distributions can be obtained by the study of literature (Giuffrè 1993, Sorrentino 2014) or historical documents, defining both the rule of art and the dimensional relationships between structural elements (Rondelet 1831, Breymann 1845). In a second time, new collected data can be used in order to refine the posterior pdf.

The adopted method aims calculating the horizontal loads multiplier $\alpha$ for walls overturning local mechanism of collapse. Obviously, obtained results are not $\alpha$ single values, but probability density functions.

For the implementation of the procedure, input (certain) data refers to masonry mechanical characteristics, building total height and number of floors. Wall thicknesses is derived, considering two different conditions:

-known interstory heights;

-unknown interstory heights.

In the second case, the analysis must be conducted introducing compositional data approach (Aitchison 1982a,b). The choice of calculating wall thicknesses and interstory heights is linked to the limited availability of on-site data obtained during rapid external survey. The decision has been validated by means of some sensitivity analysis (i.e. Taffarel et al. 2016), carried out to evaluate the relationship between geometrical parameters and $\alpha$ coefficient. As expected, the study identified only minimal coefficient variations. This situation do not indicate a poor influence of geometrical parameters on final results, but rather highlight the necessity of evaluating the integration between all possible variables, in order to obtain more realistic coefficient variations. Because of this, further developments will be focused on the study of other characteristics such as wall lengths, percentage of façade openings and floors typology and warping. Some studies consider the analysis of samples whose sum is the integer number 1 (eg. data representing soils composition). Same considerations can be applied to interstorey heights: given buildings total height and number of floors, interstorey heights can be derived, considering that their sum must correspond to buildings total height.

\section{APPLICATION OF THE APPROACH TO TIMISOARA CASE STUDY}

Abovementioned procedure is implemented considering Timisoara (Romania) historical center case study.

In 2014 an on-site survey was carried out in order to collect information about the entire set of masonry buildings located in its historical city center (Figs 12). In this paper 37 two-story masonry buildings belonging to the same typology, characterized by homogeneous vertical structures (solid brick masonry 
with lime mortar), are considered. In this context, the availability of a huge number of data is fundamental in order to validate the procedure.

The computational procedure is carried out by adopting the R Software (R DCT 2008). The code aims at calculating $\alpha$ values, defining the first step in calculation and verification of local mechanisms of collapse analysis. The decision of calculating $\alpha$ coefficient instead of other related parameters (such as spectral acceleration or participant mass) depends on the necessity to evaluate, step by step, the procedure effectiveness. Additional research developments will be focused on the analysis of above cited parameters. Because of the impossibility to evaluate numerous parameters by means of a rapid external survey, the process is implemented in a simplified formulation, without considering the containment effects linked to horizontal structures or to the presence of tie-rods. Once the procedure is validated, it will be possible to extend the study to other local mechanisms of collapse and add new parameters.

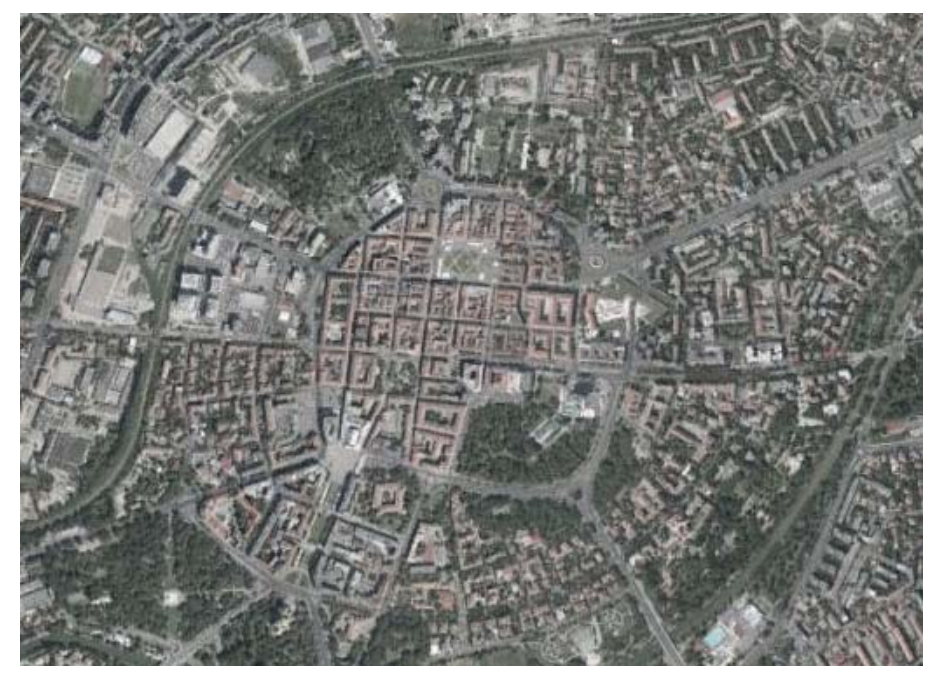

Figure 1. Aerial photo of Timisoara historical city centre (Google Earth)

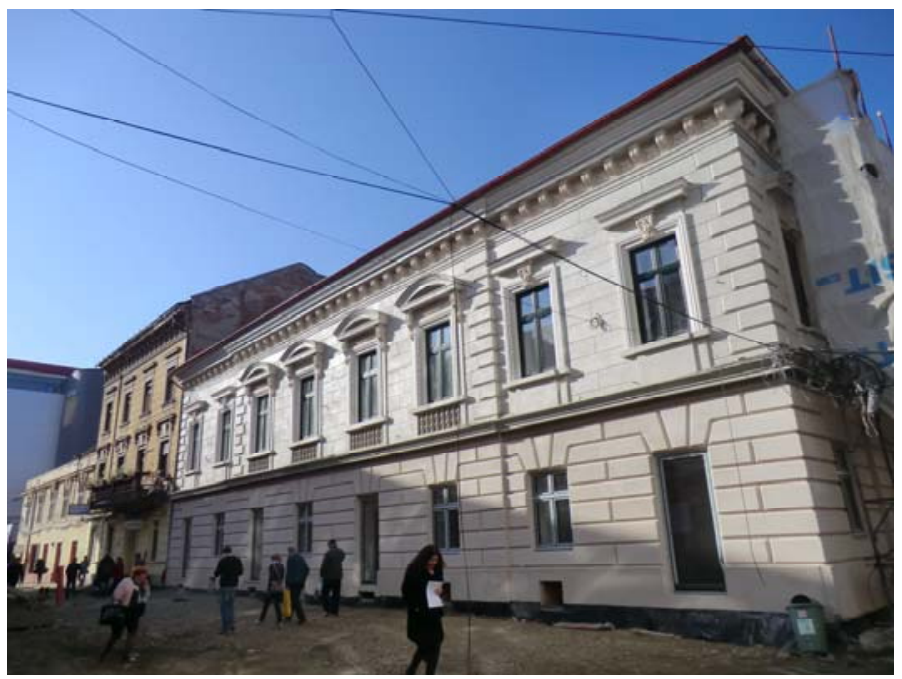

Figure 2. Aggregate buildings of Timisoara historical centre

\subsection{Bayesian updating: first evaluations concerning the refinement of prior distribution}

First, acquired information can be used in order to determine the prior distribution and to update it, defining the new posterior distribution. The influence of the number of samples adopted for the prior distribution definition is evaluated and compared to the number of data adopted for the Bayesian updating. Considering data availability (information about 37 buildings), various tests have been carried out observing if resulting $\mu$ and $\sigma$ vary increasing data adopted for definition of the prior pdf and decreasing those adopted for the posterior pdf, and vice versa. Inserted data refer to:

$$
y=\left[\begin{array}{lll}
y_{1} & \ldots & y_{n}
\end{array}\right]=\left[\log \left(\frac{h_{1}}{h_{t o t}}\right) \quad \ldots \quad \log \left(\frac{h_{n}}{h_{t o t}}\right)\right]
$$

where: $h_{1 \ldots} h_{n}=$ interstorey height of the first floor; $\mathrm{h}_{\text {tot }}=$ total height; $\mathrm{n}=$ number of floors.

Regardless the meaning of entered data, they are analyzed as pure numbers, considering the aim of this first study. Various cases are analyzed, varying input and output samples. The number of adopted samples for prior and posterior distributions does not affect significantly posterior distribution. In light of these considerations, the prior pdf (setting model) can be fixed by adopting a limited number of observations. Any new acquired information can then be used for the Bayesian updating.

Another interesting consideration refers to the trend of $\sigma$ values in posterior distribution (Fig. 3).

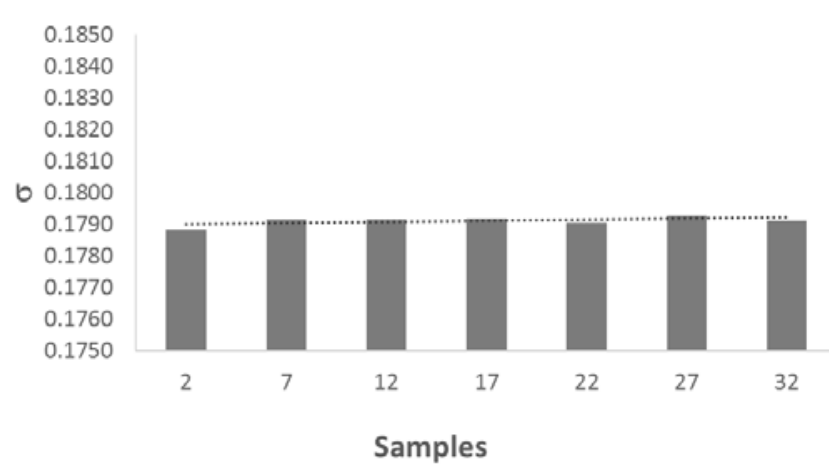

Figure 3. Trend of obtained $\sigma$ values for posterior distributions

In this case, $\sigma$ variation is not relevant (range between 0.1791 and 0.1788 ). It may be therefore appropriate to adopt a posterior distribution in which $\sigma$ value is fixed and inference only involves $\mu$ variable. In this case the procedure would be further simplified and Equation 3 can be adopted instead of Equation 4, reducing the computational burden. 


\subsection{Horizontal loads multiplier $(\alpha)$ calculation: results comparison and considerations about procedure calibration}

As previously described, the final procedure aims performing the calculation of $\alpha$ coefficient. Three different procedures are implemented, and results are compared to $\alpha$ real values obtained by adopting the standard procedure (analysis of freestanding wall overturning by using traditional spreadsheets) and by introducing data directly measured during on-site surveys. All three codes allow the calculation of $\alpha$ value for each considered freestanding wall, requiring the insertion of input data referred to:

-masonry mechanical parameters (all buildings are made of bricks; the adopted level of knowledge is LC1 as defined by the Italian code (Circolare n. 617/2009, § C8A.1.A.4)); -number of building floors;

-total building height.

The main difference between the codes is that interstory heights (where: $\mathrm{h}_{1}=$ groundfloor; $\mathrm{h}_{2}=$ first floor) of analyzed walls can be entered manually (by using real values, directly measured on-site) or can be deducted from the total building height by adopting the compositional data approach.

Regardless the use of the compositional data approach, $\mathrm{R}$ code defines the ground floor and the first floor wall thicknesses through the adoption of the Bayesian method. Starting from a limited number of data ( 5 buildings) relating to $\mathrm{s}_{2} / \mathrm{h}_{2}$ ratio $\left(\mathrm{s}_{2}=\right.$ first floor wall thickness; $h_{2}=$ first floor height) directly measured on site, a prior distribution is created. By adopting the inference process, new data related to a larger buildings sample ( 32 buildings) are inserted in the code, updating information provided in the prior distribution and creating an updated posterior distribution. By using Monte Carlo simulations, a defined number of samples related to $s_{2} / h_{2}$ ratio is extracted.

The same procedure can be adopted for $\Delta s$ values, defining the difference between the first floor and the ground floor thicknesses, or for $s_{1}$ by adopting the same procedure implemented for $s_{2} / h_{2}$ ratio (Fig. 4). $\mathrm{s}_{2}$ values can be obtained by multiplying obtained ratios for $\mathrm{h}_{2}$ value, which can be defined by using the compositional data approach or by inserting manually the measured value. The probabilistic approach assumes that the case in which the first floor wall thickness is bigger than the ground floor one could occur. In the same way, this situation has a low probability to occur. Moreover, the generation of numerous samples and the calculation of their average value allow stabilizing data in proportionally realistic intervals. This entails that the condition $\mathrm{s} 1 \geq \mathrm{s} 2$ is always verified.
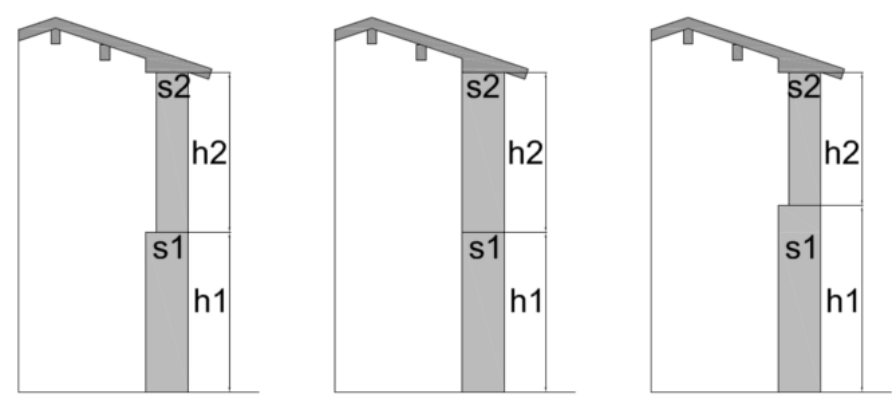

Figure 4. Example of models generated by adopting output data

Once output data are obtained, $\alpha$ value can be calculated by using formulas defined by the Italian code (D.M. 14/01/2008, Circolare n.617/2009).

Results obtained by adopting the abovementioned methods are then compared in order to evaluate the effectiveness of the probabilistic method:

1. $\alpha$ real value, calculated using the traditional method; all data are directly surveyed onsite;

2. $\alpha$ value, calculated by adopting $\mathrm{R}$ code without using the compositional data approach; Bayesian inference for the definition of $s_{2}$ and $\Delta \mathrm{s}$ values;

3. $\alpha$ value, calculated by adopting $\mathrm{R}$ code and using the compositional data approach for $\mathrm{h}_{1}$ and $\mathrm{h}_{2}$; Bayesian inference for the definition of $\mathrm{s}_{2}$ and $\Delta \mathrm{s}$ values;

4. $\alpha$ value, calculated by adopting $\mathrm{R}$ code and using the compositional data approach for $\mathrm{h}_{1}$ and $\mathrm{h}_{2}$; Bayesian inference for the definition of $s_{2}$ and $s_{1}$ values.

Obviously, the adoption of a probabilistic approach allows deriving probability distributions for $\alpha$ values and not single values. Following comparisons are performed using $\alpha$ mean value (Figs 5-6).

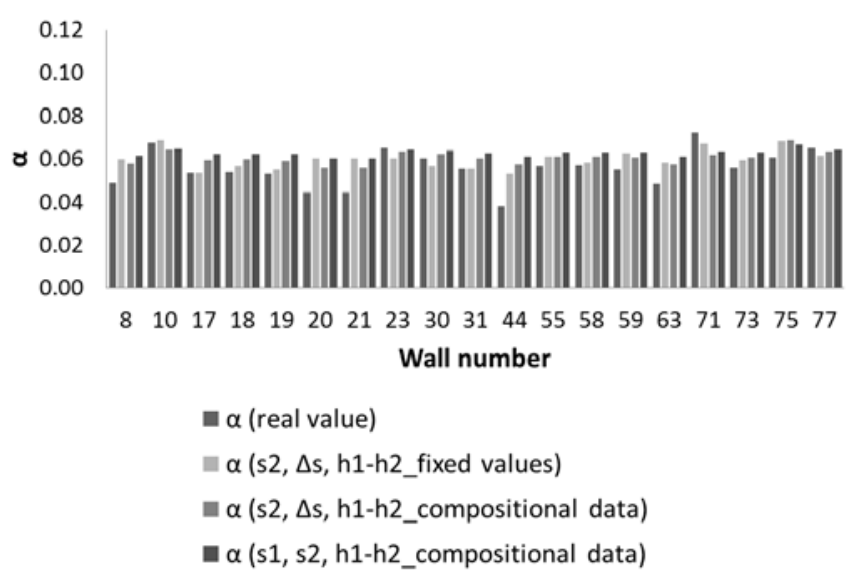

Figure 5. Comparisons between $\alpha$ values (part 1) 


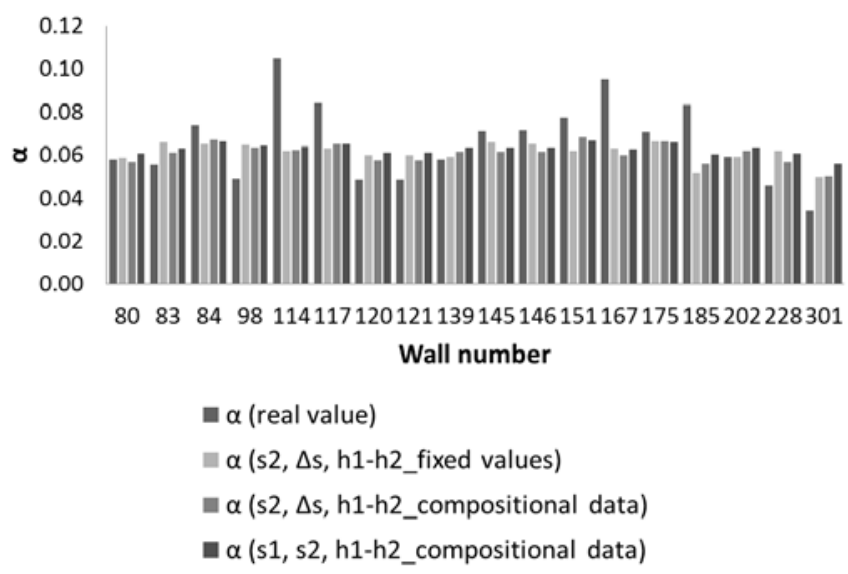

Figure 6. Comparisons between $\alpha$ values (part 2)

In order to clarify the results analysis, all obtained values for each case has been normalized with reference to their mean value.

Results obtained in case 4 show a certain homogeneity. Values tend to stabilize close to the mean value, in a small percentage range between $-0.7 \%$ and $0.4 \%$. The parameters variation with respect to case 3 is uniquely linked to $s_{1}$ calculation, which is obtained using $\mathrm{s} 1 / \mathrm{h} 1$ ratio (case 4) rather than the sum of $s_{2}$ and $\Delta s$. The code tends to equalize $s_{1}$ and $s_{2}$ values, significantly reducing $\Delta \mathrm{s}$ values compared to real cases.

Comparing case 2 and case 3 , in which the main difference is related to the generation of interstory heights by adopting the compositional data approach, results variations are not significant considering that in both cases they are included in a small range between $-1.0 \%$ and $0.8 \%$. The compositional data method shows good results. In light of analysis purposes and aiming to reduce or even avoid on-site surveys (which are an essential step in vulnerability analyses in order to collect data about interstory heights), the compositional data approach can be adopted. The collection of buildings geometric information can be limited to buildings total height and number of floors. Survey activities can then be reduced in favor of the adoption of more expeditious practices, such as analysis of aerial photos or online maps (Fabris et al. 2013).

By observing Figures 5-6, real values having a minor correspondence to computed ones refer to cases in which real geometrical features deviate from average values measured for Timisoara historic center. Results obtained by adopting the probabilistic approach can be in favor of safety or not in relation to geometrical configuration variations, which may cause an increase or a reduction in stabilizing moment. On the basis of obtained results and further validation in progress, a next step will be the definition of corrective coefficients to adopt if new parameters vary significantly compared to input ones (e.g. analyzed building wall is much thicker than previously surveyed ones). Probabilistic approaches usually represent correctly common configurations to the detriment of exceptional cases. It is however clear that exceptional cases should usually be considered and analyzed separately.

\subsection{Horizontal loads multiplier $(\alpha)$ calculation: identification of conservative values}

As previously described, the adoption of a probabilistic approach allows to obtain a probability distribution for each $\alpha$ value. For the purposes of local mechanisms of collapse analysis, it is however necessary to define a single $\alpha$ value which has to be adopted in order to carry out seismic verifications. At present, there are no parameters allowing the adjustment of $\alpha$ values in order to adapt them to real survey conditions (as it happens by adopting the Confidence Factors defined by the Italian code Circolare n.617/2009). For this reason, a first attempt to identify a selection criterion for $\alpha$, based on the comparison between real calculated $\alpha$ values and probability distributions obtained with $\mathrm{R}$ code (described at point 3 of the previous section), has been defined by taking into consideration a conservative approach. The first step of the procedure requires the standardization of $\alpha$ real values calculated for the 37 buildings of Timisoara historical center, i.e. their positioning on a normal standard distribution $(\mu=0$, $\sigma=1)$ according to the formula:

$Z=\frac{x-\mu}{\sigma}$

where $Z=$ standardized $\alpha$ value, $x=\alpha$ real value, $\mu=$ mean of the normal distribution obtained for each single building by implementing $\mathrm{R}$ code and $\sigma=$ standard deviation of the normal distribution obtained for the building population by implementing $\mathrm{R}$ code.

As indicated in Figure 7, 84\% of $\alpha$ values (31 cases out of 37) is greater than $\mu-\sigma=-1$. As a consequence, the choice of an $\alpha$ value which is smaller or equal than the difference between mean and standard deviation of each normal distribution calculated with $\mathrm{R}$ code can be considered adequate and the implementation of seismic verifications by adopting this value can be considered suitably conservative. 


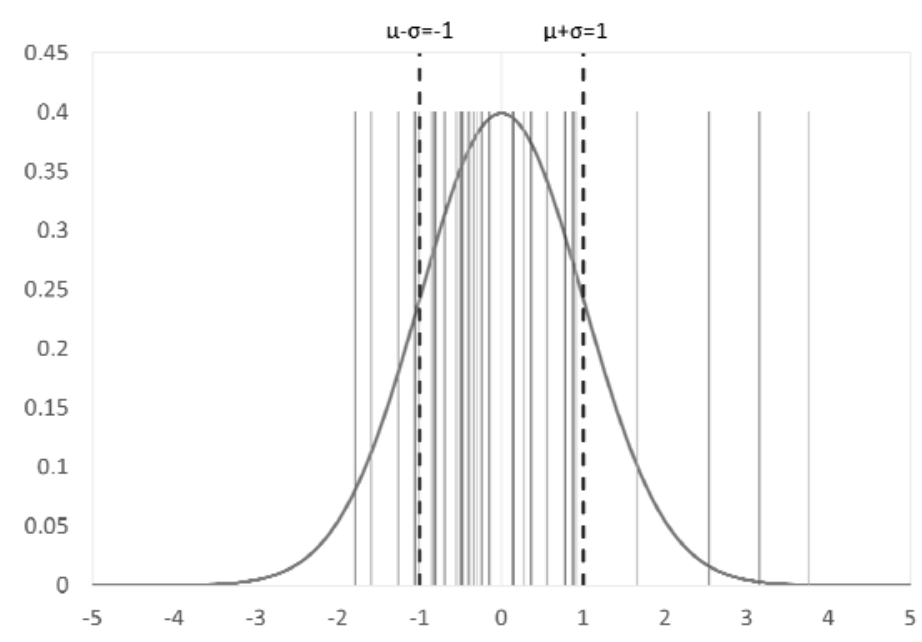

Figure 7. Normal standard distribution of $\alpha$ values. Vertical lines represent $\alpha$ real values calculated for the 37 buildings

\section{CONCLUSIONS}

This paper aims defining a new procedure for the seismic vulnerability assessment on a territorial scale through the adoption of a probabilistic approach instead of traditional deterministic methods.

The procedure enables vulnerability assessments of entire urban areas through the knowledge of a limited number of basic information, obtainable through limited on-site surveys. The data collection process can be executed through an incremental approach, in which vulnerability assessments will be refined by increasing the number of available information for the studied area.

The methodology is still at a calibration and validation stage, but the presented preliminary results confirm its reliability. Further applications will be performed, extending the procedure of local mechanisms of collapse analysis to nonlinear verifications, and increasing the number of parameters involved in the evaluation.

\section{REFERENCES}

Aitchison, J. 1982a. A Concise Guide to Compositional Data Analysis. Available on-line: http://www.leg.ufpr.br/doku.php.

Aitchison J. 1982b. The Statistical Analysis of Compositional Data in Journal of the Royal Statistical Society. Series B (Methodological), Vol. 44, No. 2. (1982), pp. 139-177.

Breymann G. A. 1845. Trattato generale di costruzioni civili. Milano: Vallardi (ed.).

Fabris, M., Achilli, V., Campostrini, G. P., Modena, C. 2013. L'aerofotogrammetria digitale per la stima delle caratteristiche strutturali degli edifici. In A.N.I.D.I.S 2013, XV convegno - L'ingegneria Sismica in Italia, Padova, Italy, 30 June -4 July 2013.

Giuffrè, A. 1993. Sicurezza e conservazione dei centri storici: il caso Ortigia. Roma-Bari: Laterza (ed.), 132.

Hoff, P. D. 2009. A First Course in Bayesian Statistical Methods. Springer (ed.).
Jackman, S. 2009. Bayesian Analysis for the Social Sciences WILEY (ed.).

Liseo, B. 2008. Introduzione alla statistica Bayesiana, Springer ed. [In Italian]

Ministero dei beni e delle Attività Culturali e del Turismo MIBACT 2015. Circular n.15 (30/04/15). Provisions on the protection of architectural heritage and seismic risk mitigation [In Italian]

Ministero dei Beni e delle Attività Culturali. 2011. Direttiva del Presidente del Consiglio dei Ministri (09/02/11). Valutazione e riduzione del rischio sismico del patrimonio culturale con riferimento alle Norme tecniche per le costruzioni di cui al D.M. 14/01/2008.

Ministero delle Infrastrutture e dei Trasporti. 2008. D.M. 14/01/2008. Nuove norme tecniche per le costruzioni.

Ministero delle Infrastrutture e dei Trasporti. 2009. Circolare esplicativa 02/02/2009 n. 617. Istruzioni per l'applicazione delle «Nuove norme tecniche per le costruzioni». D.M. $14 / 01 / 2008$.

Modena, C. 2014. Una proposta operativa di approccio sostenibile alla valutazione e mitigazione del rischio sismico dei centri storici. In I quaderni della Soprintendenza per $i$ Beni architettonici paesaggistici per le provincie di Verona Rovigo Vicenza, vol. VI, 2014.

R Development Core Team DCT. 2008. R: A language and environment for statistical computing. R Foundation for Statistical Computing, Vienna, Austria. ISBN 3-900051-07-0, URL http://www.R-project.org.

Rondelet J. 1802 - 1817. Traité théorique et pratique de l'art de bâtir. Paris.

Sorrentino, L. 2014. Reconstruction plans after the 2009 L'Aquila earthquake. From building performance to historical centre performance. In SAHC2014 - 9th International Conference on Structural Analysis of Historical Constructions, Mexico City, Mexico, 14-17 October, paper 11-006.

Taffarel S., Caliman M., Valluzzi M.R., da Porto F. \& Modena C. 2016. Seismic vulnerability assessment of clustered historical centers: fragility curves based on local collapse mechanisms analyses. In IB2MAC2016 16TH INTERNATIONAL BRICK AND BLOCK MASONRY CONFERENCE, "MASONRY IN A WORLD OF CHALLENGES", Padova, Italy, 26 - 30 June 2016. [under review] 